\title{
Flattening filter free stereotactic body radiation therapy for lung tumors: outcomes and predictive factors
}

\author{
Thitiporn Jaruthien, Sarin Kitpanit, Danita Kannarunimit, Chonnipa Nantavithya, Anussara Prayongrat, \\ Petch Alisanant, Kitwadee Saksornchai, Napapat Amornwichet, Tassapong Raiyava, \\ Chakkapong Chakkabat, Chawalit Lertbutsayanukul, Chonlakiet Khorprasert, Kanjana Shotelersuk
}

Division of Radiation Oncology, Department of Radiology, Faculty of Medicine, Chulalongkorn University, King Chulalongkorn Memorial Hospital, Thai Red Cross Society, Bangkok, Thailand

Contributions: (I) Conception and design: S Kitpanit, D Kannarunimit, T Jaruthien; (II) Administrative support: None; (III) Provision of study materials or patients: All authors; (IV) Collection and assembly of data: T Jaruthien, S Kitpanit; (V) Data analysis and interpretation: T Jaruthien, S Kitpanit, D Kannarunimit, C Nantavithya; (VI) Manuscript writing: All authors; (VII) Final approval of manuscript: All authors.

Correspondence to: Danita Kannarunimit. Division of Radiation Oncology, Department of Radiology, Faculty of Medicine, Chulalongkorn University, King Chulalongkorn Memorial Hospital, 1873 Rama IV Rd., Pathumwan, Bangkok 10330, Thailand. Email: danitakann@gmail.com.

Background: Stereotactic body radiation therapy (SBRT) using flattening filter free (FFF) has been commonly used, however, its outcomes and predictive factors in lung tumors are limiting. Thus, we aim to assess the clinical outcomes of this approach and identify factors associated with outcomes in patients with early stage non-small cell lung cancer (NSCLC) and oligometastatic/oligoprogressive lung tumor (OLT).

Methods: Patients who underwent lung SBRT with FFF were retrospectively reviewed. All patients were delivered using volumetric modulated arc therapy (VMAT) technique. The primary outcome was local control (LC). The secondary outcomes were overall survival (OS) and toxicities. We assessed the association between LC and various factors in OLT.

Results: From February 2014 to July 2019, ninety-four patients and 129 lesions with median follow-up time of 30 months were included in the analysis. Twenty-six patients with 26 lesions were early NSCLC, while 68 patients with 103 lesions were OLT, $41.7 \%$ of which were from colorectal cancers (CRC) and $18.5 \%$ were from primary lung cancers. Two-year LC was $88.9 \%$ and $85.7 \%$ for early NSCLC and OLT, respectively. Two-year OS was significantly higher for early NSCLC than OLT (83.3\% vs. 68.7\%, $\mathrm{P}=0.035)$. In the multivariate analysis for OLT, CRC origin (hazard ratio, HR 10.59, 95\% CI: 2.29-48.95, P=0.003) and gross tumor volume (GTV) mean $\mathrm{BED}_{10} \leq 147$ Gy (HR 5.16, 95\% CI: 1.13-23.59, P=0.034) were significantly associated with higher local failure (LF). Most of the acute grade 1-2 toxicities were radiation pneumonitis (26.5\%). No grade 3-5 event was observed.

Conclusions: This study confirmed the clinical efficacy and safety of lung SBRT using FFF-technique. Our findings support the role of using a high $\mathrm{BED}_{10}$ regimen to achieve good LC for OLT and the potential role for dose escalation for primary CRC.

Keywords: Stereotactic body radiation therapy (SBRT); flattening filter free (FFF); non-small cell lung cancer (NSCLC); oligometastatic/oligoprogressive lung tumor (OLT)

Submitted Oct 29, 2020. Accepted for publication Jan 06, 2021.

doi: $10.21037 /$ tcr-20-3174

View this article at: http://dx.doi.org/10.21037/tcr-20-3174 


\section{Introduction}

Unlike conventional radiation therapy, stereotactic body radiation therapy (SBRT) allows accurate delivery of a highly focused ablative radiation dose to the tumor in fewer treatment fractions while reducing unnecessary dose to the surrounding normal tissues. Currently, SBRT is considered a treatment option for early stage non-small cell lung cancer (NSCLC) patients who are medically inoperable or deny surgery with excellent outcome (1-3). For patients with lung metastases, reports on the use of SBRT in patients whose metastases are limited in number (oligometastases) or limited by site of progression (oligoprogression) are emerging and the results are promising $(4,5)$.

Flattening filter is generally used to provide flat dose at a certain depth to give uniform intensity across the treatment field. The removal of flattening filter allows the delivery of the flattening-filter-free (FFF) beam contributes to dose rate escalation. FFF beam has a cone-shaped dose profile, giving up to four-fold higher dose rate in the center of the beam compared to the flattened beam (Figure 1). This FFF characteristics facilitate treatment optimization to shorten treatment time, sharper penumbra and less out-of-field dose that should translate into less intrafraction motion error and less dose to normal tissue (6-8). Despite numerous dosimetric studies, there are few clinical studies on SBRT with FFF in early stage NSCLC and lung metastases that have been reported (9-14). Therefore, we retrospectively assessed the 2-year local control (LC), overall survival (OS) and predictive factors relating to the treatment outcomes in patients who received lung SBRT using FFF. We present the following article in accordance with the STROBE reporting checklist (available at http://dx.doi.org/10.21037/tcr-20-3174).

\section{Methods}

\section{Study population}

Our study is a retrospective, single center study. This study was approved by the Institutional Review Board of the Faculty of Medicine, Chulalongkorn University, Bangkok, Thailand (approval No. 465/60) and conducted in accordance with the Declaration of Helsinki (as revised in 2013). Because of the retrospective nature of the research, the requirement for informed consent was waived. After receiving the approval from the institutional review board, we retrospectively identified and selected patients with early stage NSCLC and oligometastatic/oligoprogressive lung tumor (OLT) treated with SBRT using FFF at King Chulalongkorn Memorial
Hospital from February 2014 to July 2019. Oligometastases was defined as up to 3 metastases in any single organ system and up to 5 hematogenous metastases with controlled primary tumor. Oligoprogression was defined as the progression of a solitary or few tumors in the lung while the rest of the tumors responded to or were stable with systemic therapy. Patients with follow-up period less than 3 months or lacked follow-up imaging were excluded.

\section{Treatment and evaluation}

Four-dimension computed tomography (4D-CT) or deep inspiration breath hold (DIBH) CT simulation were used to account for internal organ motion. Axial acquisitions were done with spacing $\leq 3 \mathrm{~mm}$. Gross tumor volume (GTV) was delineated using CT pulmonary window. No expansion was added to create clinical target volume (CTV). An additional $0.5 \mathrm{~cm}$ radially and $1 \mathrm{~cm}$ craniocaudally were added to GTV to create planning target volume (PTV) for patients using breath hold techniques, while an additional $0.5 \mathrm{~cm}$ in all dimensions was added to create PTV for patient using 4D-CT. All patients received unflattened beam using 6 and $10 \mathrm{MV}$ photon with volumetric modulated arc therapy (VMAT) technique on Varian Eclipse ${ }^{\mathrm{TM}}$ Treatment Planning System version 11.0.31 (Varian Medical Systems, Palo Alto, USA). The maximum dose rate for FFF beams was 1,400 monitor units (MU)/min for $6 \mathrm{MV}$ and 2,400 MU/min for $10 \mathrm{MV}$. Dose fractionation and plan evaluation followed our institution's protocol. Early stage NSCLC received radiation dose ranged from 48-60 Gy in 3-8 fractions based on tumor location. For lung metastases, the dose was selected from the protocol or at the discretion of the treating physicians. Dose was prescribed to isodose between $60-90 \%$ where the center of mass was normalized to $100 \%$. Treatment planning goals included $100 \%$ prescription dose delivered to $95 \%$ of the PTV. Dose to critical structures was achieved according to American Association of Physicists in Medicine (AAPM) Task Group 101 recommendation (15). The interval between treatment fraction was $24-48$ hours.

Post-treatment evaluation included clinical examination, CT scan of the thorax, and/or fluorodeoxyglucose-positron emission tomography (FDG-PET) CT scan at 3 and 6 months after SBRT, every 3-6 months for the first 2 years, and then every 6 months for up to 5 years, and annually thereafter. Tumor response was officially evaluated by radiologists.

The primary outcome of the study was LC. LC was defined as stable disease, partial or complete response as 
A

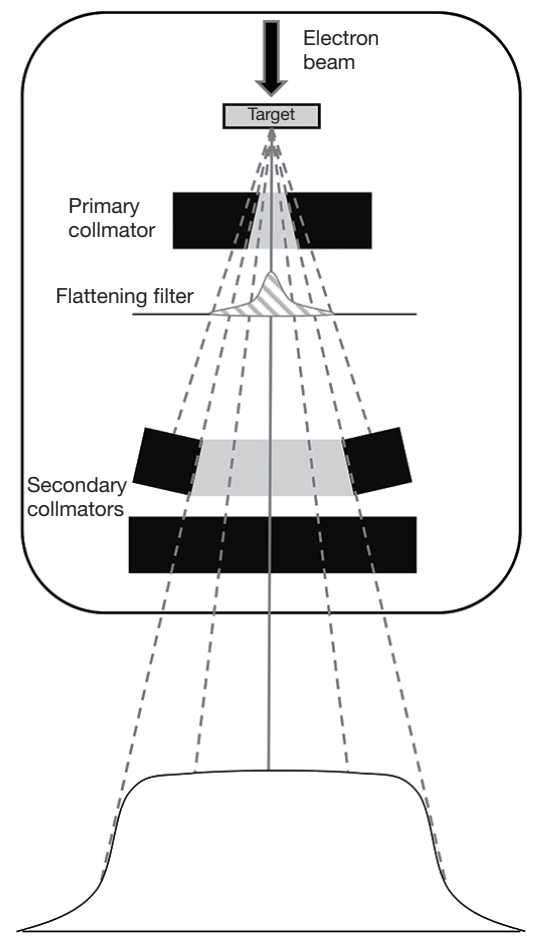

B

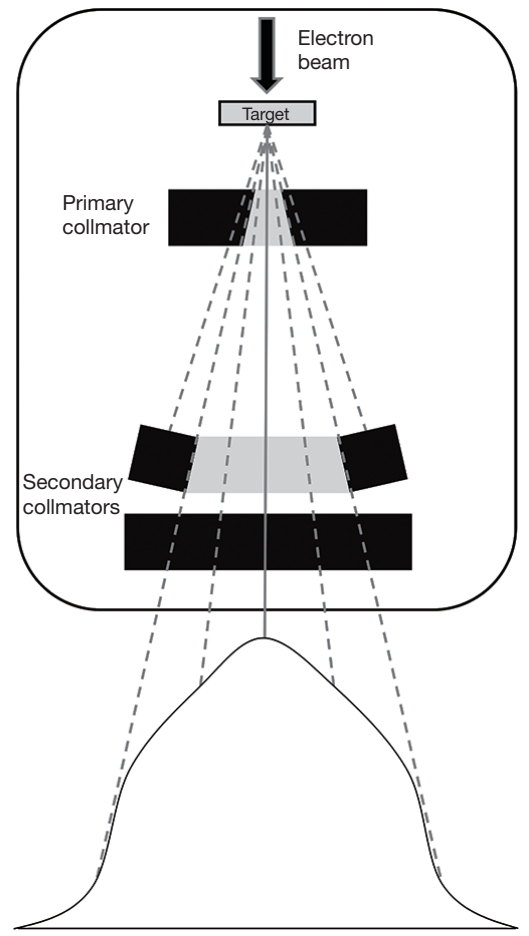

Figure 1 Shows beam profiles obtained using flattened filter (A) and flattening filter free (B).

observed at follow-up imaging and determined by using Response Evaluation Criteria in Solid Tumors (RECIST) criteria version 1.1. All targeted lesions were accounted for LC assessment. Patients were observed for LC, even if new distant lesions were found. The secondary outcomes were OS, and early and late toxicities. Time to LC and survival were assessed from the date of the first SBRT. Patterns of failure included local failure (LF), regional failure, involved lobe failure and distant failure were recorded. LF was defined as recurrence within irradiated PTV areas. Regional failure was defined as recurrence at the regional lymph node within the lung, bronchial hilum or mediastinum. Involved lobe failure was defined as recurrence in the same lobe. Distant failure was defined as a failure outside the primary lobe and at other organs. When biopsy was not possible, the radiographic progression was defined as an interval increase in size of mass or focal metabolic uptake on FDG-PET/CT. Data were extracted from the patient's medical records and treatment planning system. All doses were converted into biological effective dose at $\alpha / \beta=10\left(\mathrm{BED}_{10}\right)$ using linearquadratic model: $\mathrm{BED}_{10}=$ number of fractions $\mathrm{x}$ dose per fraction $(1+$ dose per fraction/10). Acute and late toxicities were graded according to the Common Terminology Criteria for Adverse Events (CTCAE) version 4.03.

\section{Statistical analysis}

LC and OS were assessed using the Kaplan-Meier method. Log rank test was used to compare survival between 2 groups. Cox regression model was used for univariate analysis to identify the relationship between the outcomes and the prognostic factors. Factors with $\mathrm{P}$ values of $<0.2$ were incorporated into the multivariate logistic regression model using a stepwise backwards selection process. The cut-off values for dose-volume parameters were determined using the receiver operating characteristic (ROC) analysis and its area under the curve (AUC). Youden's index was used to identify the cut-off values that gave the maximum sensitivity and specificity. The SPSS software (version 22.0; IBM) was used for the statistical computations. All tests were two-sided and $\mathrm{P}$ values of less than 0.05 was considered statistically significant.

\section{Results}

\section{Patients, tumors, and treatment characteristics}

Between February 2014 to July 2019, two from 96 patients were excluded due to lack of follow-up imaging after treatment. The remaining 94 patients with 129 lesions 
were included for analysis with median follow-up time of 30 months (interquartile range, 16-43 months). Patient and tumor characteristics are shown in Table 1. Median age of all patients at time of SBRT was 65 years (IQR 55-75 years). Of 129 lesions, $20.2 \%$ were early NSCLC and $79.8 \%$ were lung metastases. The most common primaries of the lung metastases were colorectal cancer (CRC) (41.7\%), NSCLC (18.4\%) and head and neck cancer (14.6\%). Indication for SBRT in patients with early stage NSCLC was mainly medically inoperable (92.3\%). Doses of 48-60 Gy in 3-8 fractions (7.5-18 Gy/fraction, BED 100-151.2 $\mathrm{Gy}_{10}$ ) were given to early NSCLC and 25-70 Gy in 1-10 fractions (5-30 Gy/fraction, BED 48-180 $\mathrm{Gy}_{10}$ ) were given to lung metastases. The most used dose fractionation was $12 \mathrm{~Gy}$ $\times 5$ fractions for early NSCLC and 10 Gy $\times 5$ fractions for metastatic lung cancers. All patients were treated with VMAT with FFF technique and completed treatment without any interruption.

\section{Outcomes}

At the final analysis, two lesions from the early stage NSCLC group and 13 lesions from the lung metastases group had LF. Six patients (13.6\%) from the early NSCLC group and 36 patients $(52.9 \%)$ from the lung metastases group died. Two-year LC was achieved in $88.9 \%$ and $85.7 \%$ in patients with early NSCLC and OLT, respectively; the 2 -year LC was comparable between the 2 groups $(\mathrm{P}=0.464)$. Two-year OS was significantly higher for early NSCLC; the 2-year OS for early NSCLC was $83.3 \%$ and OLT was $68.7 \%(\mathrm{P}=0.035)$ (Figure 2). Median OS was 38 months for OLT. Distant failure was the predominant pattern of failure for both groups as shown in Table 2. The most common toxicities were radiation pneumonitis $(22.3 \%$ had grade 1 , and $4.2 \%$ had grade 2$)$ followed by chest wall pain $(4.3 \%$ had grade 1 , and $2.1 \%$ had grade 2 ). No acute and late toxicity greater than grade 2 were found. There was no difference between central and peripheral tumor groups regarding toxicities except fracture of the rib which was found in 7 patients $(7.4 \%)$ with extreme peripheral lesions.

\section{Factors predicting local recurrence after SBRT for oligometastases/OLTs}

Due to the small number of early stage NSCLC patients, we did not perform the univariate analysis to define factors associated with outcomes. For the metastases group, the univariate analysis showed that the age, sex, primary tumor origin, tumor maximal diameter, tumor location and radiation dose, including prescribed dose $\mathrm{BED}_{10}$ (cut off $100 \mathrm{~Gy}$ ), PTV mean BED 10 (cut off $130 \mathrm{~Gy}$ ), $\mathrm{BED}_{10}$ to $95 \%$ of PTV volume (D95) (cut off 108 Gy) and GTV mean $\mathrm{BED}_{10}$ (cut off $147 \mathrm{~Gy}$ ), were associated with LF. However, in multivariate analysis, only CRC origin [HR 10.59, $95 \%$ confidential interval $(\mathrm{CI}), 2.29-48.95, \mathrm{P}=0.003]$ and lower GTV mean $\mathrm{BED}_{10}$ (HR 5.16, 95\% CI: 1.13-23.59, $\mathrm{P}=0.034$ ) were significant predictors for higher LF (Table 3).

In the lung metastatic group, 43 lesions were primary CRC and 11 lesions had LF. All dose parameters except for prescribed $\mathrm{BED}_{10}$ were significantly associated with $\mathrm{LF}$. GTV mean $\mathrm{BED}_{10} \leq 147$ Gy, PTV mean $\mathrm{BED}_{10} \leq 130$ Gy and, PTV D95 BED $_{10} \leq 108$ Gy were associated with an increase in LF in CRC metastases (Figure 3). There was no significant difference in OS regardless of recurrent status $(\mathrm{P}=0.226)$ or primary histology $(\mathrm{P}=0.434)$.

\section{Discussion}

This study confirmed the efficacy of FFF-VMAT for SBRT in the treatment of lung cancers with no severe toxicity. The results were consistent with previous studies reporting the clinical outcomes of SBRT which had a 2-year LC of 87.9$98.5 \%$ in early NSCLC and $77.9-88 \%$ in metastatic lung tumor $(1,5,16-22)$. Our results were also in line with other studies investigating SBRT with FFF in lung tumors which had a LC of $89-100 \%$ for both primary and secondary lung tumors $(9,23,24)$. FFF technique permits a considerable increase in the dose rate delivery and reduce treatment time by more than $50 \%$ compared to flattening filter (FF) technique without compromising the plan's quality $(23,25,26)$. Our study reported median beam-on-time of less than 2 minutes which was in line with previous VMATFFF studies conducted in patients with lung tumors $(12,13)$. Decreased treatment time can improve treatment efficacy for SBRT because it reduces the likelihood of intrafraction motion and reduces the likelihood of undesirable patient motion during treatment. However, the radio-biological consequence of this technique is still unknown. Few studies compared clinical outcome of FFF and FF techniques and found no difference regarding LC rate and toxicities $(13,14)$. Due to limited data of clinical efficacy and safety of FFF method, our study suggested that FFF-VMAT for SBRT shorten the treatment time while maintaining a high LC rate with low toxicity.

There were conflicting results of outcome after SBRT in lung metastases regarding primary cancer (20,27-29). 
Table 1 Patient, tumor, and treatment characteristics

\begin{tabular}{|c|c|c|}
\hline Characteristics & Early NSCLC (N=26, 26 lesions) & Lung metastases ( $\mathrm{N}=68,103$ lesions) \\
\hline Median age (range), years & $76(55.0-89)$ & $60(20.0-87)$ \\
\hline \multicolumn{3}{|l|}{ Sex, n (\%) } \\
\hline Female & $14(53.8)$ & $25(36.8)$ \\
\hline \multicolumn{3}{|l|}{ ECOG, n (\%) } \\
\hline 1 & $17(65.4)$ & $17(25.0)$ \\
\hline 2 & $5(19.2)$ & $3(4.4)$ \\
\hline \multicolumn{3}{|l|}{ Histology, n (\%) } \\
\hline Adenocarcinoma & $22(84.6)$ & - \\
\hline Colorectal cancer & - & $43(41.7)$ \\
\hline NSCLC & - & $19(18.5)$ \\
\hline Head and neck cancer & - & $15(14.6)$ \\
\hline Gynecological cancer & - & $9(8.7)$ \\
\hline Others & - & $17(16.5)$ \\
\hline \multicolumn{3}{|l|}{ T stage ( $7^{\text {th }}$ AJCC), $n$ (\%) } \\
\hline $\mathrm{T} 1$ & $12(46.2)$ & \\
\hline T2 & $14(53.8)$ & \\
\hline $2-3$ & - & $44(42.7)$ \\
\hline Median tumor diameter (range), $\mathrm{cm}$ & $3(1.3-5.7)$ & $1.9(0.6-6.4)$ \\
\hline Median GTV volume (range), cc & $15.7(2.5-176)$ & $5(0.5-226)$ \\
\hline Median PTV volume (range), cc & $46(10.2-282.7)$ & $19.2(2.1-478.4)$ \\
\hline Median no. of fractions (range) & $5(3-8)$ & $5(1-10)$ \\
\hline Median prescribed $\mathrm{BED}_{10}$ (range), Gy & $132(100-151.2)$ & $105(48-180)$ \\
\hline Median PTVD95 BED $_{10}$ (range), Gy & $134.4(102.6-163.9)$ & $104.6(49.8-193.4)$ \\
\hline Median PTV mean BED $_{10}$ (range), Gy & $162.6(126.7-201.9)$ & $128.2(58.6-231.4)$ \\
\hline Median GTV mean BED $_{10}$ (range), Gy & $178.6(133.9-225.9)$ & $145.5(63.1-282.4)$ \\
\hline Median beam on time (range), min & $1.87(1.08-2.6)$ & $1.97(0.9-9.45)$ \\
\hline
\end{tabular}

NSCLC, non-small cell lung cancer; N, number of patients; ECOG, Eastern Cooperative Oncology Group performance status; NOS, not otherwise specified; AJCC, American Joint Committee on Cancer; SBRT, stereotactic body radiotherapy; GTV, gross tumor volume; PTV, planning target volume; BED, biological effective dose. 

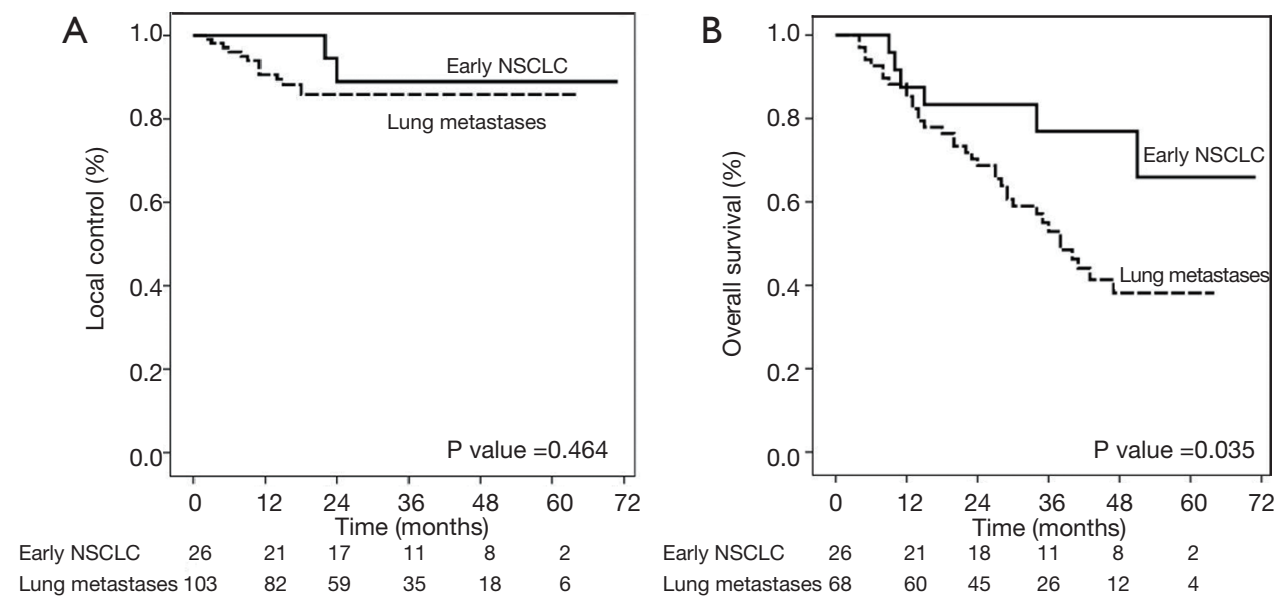

Figure 2 The Kaplan-Meier curves for local control (A) and overall survival (B) after lung SBRT. NSCLC, non-small cell lung cancer; SBRT, stereotactic body radiation therapy.

Table 2 Patterns of failure after SBRT

\begin{tabular}{lcc}
\hline Variable & Early NSCLC, N (\%) & Lung metastases, N (\%) \\
\hline Local failure & $2(7.7)$ & $13(12.6)$ \\
Regional failure & $1(3.8)$ & $12(11.7)$ \\
Involved lobe failure & $2(7.7)$ & $21(20.4)$ \\
Distant failure & $9(34.6)$ & $39(37.9)$ \\
\hline
\end{tabular}

SBRT, stereotactic body radiation therapy; NSCLC, non-small cell lung cancer; N, number of lesions.

Table 3 Univariate and multivariate analysis for local control in lung metastases group

\begin{tabular}{|c|c|c|c|c|c|c|}
\hline Factors & \multicolumn{3}{|c|}{ Univariate } & \multicolumn{3}{|c|}{ Multivariate } \\
\hline Age (years), $\leq 60$ vs. $>60$ & 3.14 & $0.86-11.41$ & 0.082 & & & \\
\hline Sex, male vs. female & 3.07 & $0.68-13.86$ & 0.145 & & & \\
\hline Primary tumor, CRC vs. others & 8.02 & $1.78-36.21$ & 0.007 & 10.59 & $2.29-48.95$ & $0.003^{\star}$ \\
\hline GTV volume, $>5$ vs. $\leq 5 \mathrm{cc}$ & 1.27 & $0.43-3.77$ & 0.671 & & & \\
\hline PTV volume, $>20$ vs. $\leq 20 \mathrm{cc}$ & 0.98 & $0.33-2.93$ & 0.977 & & & \\
\hline Location, central vs. peripheral & 2.42 & $0.75-7.87$ & 0.141 & & & \\
\hline Prescribed $\mathrm{BED}_{10}, \leq 100$ vs. $>100$ Gy & 4.26 & $1.17-15.48$ & 0.028 & & & \\
\hline GTV mean $\mathrm{BED}_{10}, \leq 147$ vs. $>147$ Gy & 5.74 & $1.27-25.92$ & 0.023 & 5.16 & $1.13-23.59$ & $0.034^{*}$ \\
\hline
\end{tabular}

*Backward stepwise regression analysis. CRC, colorectal cancer; GTV, gross tumor volume; PTV, planning target volume; BED, biological effective dose; $\mathrm{HR}$, hazard ratio; $\mathrm{Cl}$, confidential interval. 

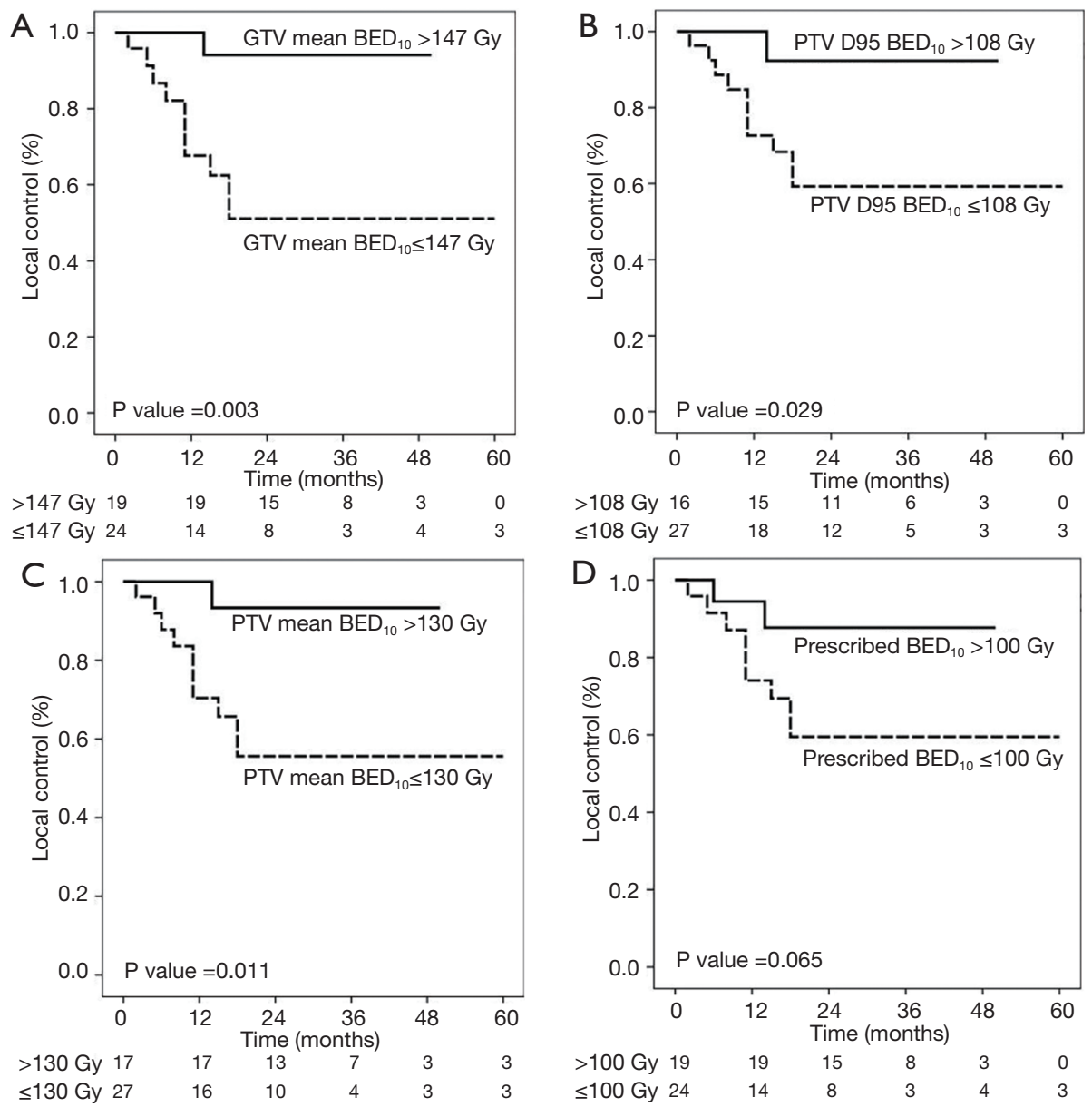

Figure 3 The Kaplan-Meier curve for local control for colorectal lung metastases with irradiation doses. GTV mean BED $10>147$ and $\leq 147$ Gy (A), PTV D95 BED $10 \leq 108$ and $>108$ Gy (B) PTV mean BED $_{10} \leq 130$ and $>130$ Gy (C), prescribed BED $10>100$ and $\leq 100$ Gy (D). GTV, gross tumor volume; BED, biological effective dose; PTV, planning target volume.

Compared to other histologies, Binkley et al. (27) treated 77 patients with 122 oligometastatic lung tumors with SBRT and reported significantly higher cumulative incidence of LF at 12 and 24 months (25.5\% and $42.2 \%$, respectively) in CRC lung metastases. Takeda and colleagues (29) also reported LC rate in CRC oligo-metastatic lung tumors was significantly worse than other origins. Similarly, we observed decreased local tumor control for CRC lung metastases but comparable OS. This is probably due to the salvageability of the metastatic lesions and/or effective systemic therapy for CRC.

Currently, there is no consensus for optimal SBRT dose for lung metastases. It has been widely accepted that a $\mathrm{BED}_{10}$ at isocenter $>100 \mathrm{~Gy}$ is required to achieve optimal LC in early NSCLC (30). The practice of SBRT for lung metastases has mostly been adapted from experiences in early NSCLC. Therefore, data of dose-response relationship in lung metastasis is lacking. A data of 327 lung metastases patients treated with SBRT from a multinational center showed that BED of $100 \mathrm{~Gy}_{10}$ or more was associated with better LC (31). However, using the prescribed dose may not be a good parameter. Even if the prescribed dose is the same, the dose received by the PTV can be different depending on the choice of prescription dose line and dose distribution. A few studies suggested that the mean dose of GTV and PTV may be relevant in predicting the outcome for lung SBRT treatment (18,32). Zhao and colleagues (18) conducted a large series to identify the optimal dose parameters for predicting local/lobar control after SBRT in early stage NSCLC and found that BED to $95 \%$ of PTV >86 Gy and PTV mean BED $10>130$ Gy were both significantly associated with decreased LF. In our 
study, average mean dose of PTV of primary lung cancer group achieved this radiation dose level, 162.6 Gy. In contrast, the mean dose of PTV of metastatic group was only 128.2 Gy. This could explain our findings that LF in metastatic group is higher than primary lung cancer group. Likewise, GTV mean $\mathrm{BED}_{10} \leq 147$ Gy was a significant predictor for LF in our study. Furthermore, we found GTV mean $\mathrm{BED}_{10} \leq 147$ Gy, PTV mean $\mathrm{BED}_{10}$ $\leq 130 \mathrm{~Gy}$, and PTV D95 BED $10 \leq 108$ Gy were associated with an increase in LF in CRC lung metastases. These results supported the idea that primary CRC was probably a radioresistant phenotype and had a dose-response relationship (33). Therefore, the current study suggested that adequate dose should be considered to achieve good LC for OLT and potential role for dose escalation for primary CRC. Recently, patterns of in-field recurrence after SABR in early stage NSCLC have been classified into central high-dose and peripheral high-dose failures which the underlying causes are possibly different (19). Clonal resistance could be the cause of central highdose failure which dose escalation is essential for better LC. Further investigation of in-field recurrence patterns in metastatic lung cancer could give insight to improve radiation treatment for better outcomes. In addition, GTV mean $\mathrm{BED}_{10}$, PTV mean $\mathrm{BED}_{10}$ and PTV D95 BED 10 should be taken into account when optimizing the SBRT plan for the treatment of lung tumors.

This study had some limitations. First, the dose fractionation in our study was not uniformly prescribed. There were a wide range of radiation doses and fractionations that were used because there is no standard fractionation for lung metastases. However, these heterogenous doses help us to determine the dose-response relationship for LC and warrant prospective clinical trials to confirm the values of this dose-outcome relationship. Second, the sample size of our early stage NSCLC group was too small to detect any relationship between the factors and outcomes. Most of the patients in our hospital were OLT. Third, all of our patients were from a single institution with short follow-up period. Therefore, additional studies with a larger sample size and longer follow-up period should assess the FFF's effects in early stage NSCLC and OLT patients.

\section{Conclusions}

This study confirms the clinical efficacy and safety of lung SBRT using FFF-technique for both early stage NSCLC and oligometastatic/oligoprogressive lung cancer patients. Our findings support the role of using a high $\mathrm{BED}_{10}$ regimen to achieve good LC for OLT and the potential role for dose escalation for primary CRC.

\section{Acknowledgments}

We would like to thank for English editing service which offered through the Research Affairs at Faculty of Medicine, Chulalongkorn University.

Funding: None.

\section{Footnote}

Reporting Checklist: The authors have completed the STROBE reporting checklist. Available at http://dx.doi. org/10.21037/tcr-20-3174

Data Sharing Statement: Available at http://dx.doi. org/10.21037/tcr-20-3174

Conflicts of Interest: All authors have completed the ICMJE uniform disclosure form (available at http://dx.doi. org/10.21037/tcr-20-3174). The authors have no conflicts of interest to declare.

Ethical Statement: The authors are accountable for all aspects of the work in ensuring that questions related to the accuracy or integrity of any part of the work are appropriately investigated and resolved. This study was approved by the Institutional Review Board of the Faculty of Medicine, Chulalongkorn University, Bangkok, Thailand (approval No. 465/60) and conducted in accordance with the Declaration of Helsinki (as revised in 2013). Because of the retrospective nature of the research, the requirement for informed consent was waived.

Open Access Statement: This is an Open Access article distributed in accordance with the Creative Commons Attribution-NonCommercial-NoDerivs 4.0 International License (CC BY-NC-ND 4.0), which permits the noncommercial replication and distribution of the article with the strict proviso that no changes or edits are made and the original work is properly cited (including links to both the formal publication through the relevant DOI and the license). See: https://creativecommons.org/ licenses/by-nc-nd/4.0/. 


\section{References}

1. Bezjak A, Paulus R, Gaspar LE, et al. Safety and efficacy of a five-fraction stereotactic body radiotherapy schedule for centrally located non-small-cell lung cancer: NRG Oncology/RTOG 0813 Trial. J Clin Oncol 2019; 37:1316-25.

2. Fakiris AJ, McGarry RC, Yiannoutsos CT, et al. Stereotactic body radiation therapy for early-stage non-small-cell lung carcinoma: four-year results of a prospective phase II study. Int J Radiat Oncol Biol Phys 2009;75:677-82.

3. Timmerman R, Paulus R, Galvin J, et al. Stereotactic body radiation therapy for inoperable early stage lung cancer. JAMA 2010;303:1070-6.

4. Alongi F, Arcangeli S, Filippi AR, et al. Review and uses of stereotactic body radiation therapy for oligometastases. Oncologist 2012;17:1100-7.

5. Siva S, MacManus M, Ball D. Stereotactic radiotherapy for pulmonary oligometastases: a systematic review. J Thorac Oncol 2010;5:1091-9.

6. Lang S, Shrestha B, Graydon S, et al. Clinical application of flattening filter free beams for extracranial stereotactic radiotherapy. Radiother Oncol 2013;106:255-9.

7. Shimozato T, Aoyama Y, Matsunaga T, et al. Beam characterization of 10-MV photon beam from medical linear accelerator without flattening filter. J Med Phys 2017;42:65-71.

8. Xiao Y, Kry SF, Popple R, et al. Flattening filter-free accelerators: a report from the AAPM therapy emerging technology assessment work group. J Appl Clin Med Phys 2015;16:5219.

9. Navarria P, Ascolese AM, Mancosu P, et al. Volumetric modulated arc therapy with flattening filter free (FFF) beams for stereotactic body radiation therapy (SBRT) in patients with medically inoperable early stage non small cell lung cancer (NSCLC). Radiother Oncol 2013;107:414-8.

10. Prendergast BM, Dobelbower MC, Bonner JA, et al. Stereotactic body radiation therapy (SBRT) for lung malignancies: preliminary toxicity results using a flattening filter-free linear accelerator operating at 2400 monitor units per minute. Radiat Oncol 2013;8:273.

11. Scorsetti M, Alongi F, Castiglioni S, et al. Feasibility and early clinical assessment of flattening filter free (FFF) based stereotactic body radiotherapy (SBRT) treatments. Radiat Oncol 2011;6:113.

12. Annede P, Darreon J, Benkemouche A, et al. Flattening filter free vs. flattened beams for lung stereotactic body radiation therapy. Anticancer Res 2017;37:5133-9.

13. Aoki S, Yamashita H, Haga A, et al. Flattening filter-free technique in volumetric modulated arc therapy for lung stereotactic body radiotherapy: A clinical comparison with the flattening filter technique. Oncol Lett 2018;15:3928-36.

14. Pokhrel D, Sanford L, Dhanireddy B, et al. Flattening filter free VMAT for a stereotactic, single-dose of $30 \mathrm{~Gy}$ to lung lesion in a 15-min treatment slot. J Appl Clin Med Phys 2020;21:6-12.

15. Benedict SH, Yenice KM, Followill D, et al. Stereotactic body radiation therapy: the report of AAPM Task Group 101. Med Phys 2010;37:4078-101.

16. Chang JY, Liu H, Balter P, et al. Clinical outcome and predictors of survival and pneumonitis after stereotactic ablative radiotherapy for stage I non-small cell lung cancer. Radiat Oncol 2012;7:152.

17. Li Q, Swanick CW, Allen PK, et al. Stereotactic ablative radiotherapy (SABR) using 70 Gy in 10 fractions for nonsmall cell lung cancer: exploration of clinical indications. Radiother Oncol 2014;112:256-61.

18. Zhao L, Zhou S, Balter P, et al. Planning target volume D95 and mean dose should be considered for optimal local control for stereotactic ablative radiation therapy. Int J Radiat Oncol Biol Phys 2016;95:1226-35.

19. Nantavithya C, Gomez DR, Chang JY, et al. An improved method for analyzing and reporting patterns of in-field recurrence after stereotactic ablative radiotherapy in early-stage non-small cell lung cancer. Radiother Oncol 2020;145:209-214.

20. Guckenberger M, Klement RJ, Allgauer M, et al. Local tumor control probability modeling of primary and secondary lung tumors in stereotactic body radiotherapy. Radiother Oncol 2016;118:485-91.

21. Rieber J, Streblow J, Uhlmann L, et al. Stereotactic body radiotherapy (SBRT) for medically inoperable lung metastases-A pooled analysis of the German working group "stereotactic radiotherapy". Lung Cancer 2016;97:51-8.

22. Singh D, Chen Y, Hare MZ, et al. Local control rates with five-fraction stereotactic body radiotherapy for oligometastatic cancer to the lung. J Thorac Dis 2014;6:369-74.

23. Rieber J, Tonndorf-Martini E, Schramm O, et al. Establishing stereotactic body radiotherapy with flattening filter free techniques in the treatment of pulmonary lesions - initial experiences from a single institution. Radiat Oncol 2016;11:80.

24. Navarria P, Ascolese AM, Tomatis S, et al. Stereotactic 
body radiotherapy (sbrt) in lung oligometastatic patients: role of local treatments. Radiat Oncol 2014;9:91.

25. Thomas EM, Popple RA, Prendergast BM, et al. Effects of flattening filter-free and volumetric-modulated arc therapy delivery on treatment efficiency. J Appl Clin Med Phys 2013;14:4328.

26. Prendergast BM, Fiveash JB, Popple RA, et al. Flattening filter-free linac improves treatment delivery efficiency in stereotactic body radiation therapy. J Appl Clin Med Phys 2013;14:4126.

27. Binkley MS, Trakul N, Jacobs LR, et al. Colorectal histology is associated with an increased risk of local failure in lung metastases treated with stereotactic ablative radiation therapy. Int J Radiat Oncol Biol Phys 2015;92:1044-52.

28. Fode MM, Hoyer M. Survival and prognostic factors in 321 patients treated with stereotactic body radiotherapy for oligo-metastases. Radiother Oncol 2015;114:155-60.

29. Takeda A, Kunieda E, Ohashi T, et al. Stereotactic body

Cite this article as: Jaruthien T, Kitpanit S, Kannarunimit D, Nantavithya C, Prayongrat A, Alisanant P, Saksornchai K, Amornwichet N, Raiyava T, Chakkabat C, Lertbutsayanukul C, Khorprasert C, Shotelersuk K. Flattening filter free stereotactic body radiation therapy for lung tumors: outcomes and predictive factors. Transl Cancer Res 2021;10(2):571-580. doi: $10.21037 /$ tcr-20-3174 radiotherapy (SBRT) for oligometastatic lung tumors from colorectal cancer and other primary cancers in comparison with primary lung cancer. Radiother Oncol 2011;101:255-9.

30. Onishi H, Shirato H, Nagata Y, et al. Hypofractionated stereotactic radiotherapy (HypoFXSRT) for stage I nonsmall cell lung cancer: updated results of 257 patients in a Japanese multi-institutional study. J Thorac Oncol 2007;2:S94-100.

31. Ricco A, Davis J, Rate W, et al. Lung metastases treated with stereotactic body radiotherapy: the RSSearch ${ }^{\circledR}$ patient registry's experience. Radiat Oncol 2017;12:35.

32. Baumann R, Chan MKH, Pyschny F, et al. Clinical results of mean GTV dose optimized robotic-guided stereotactic body radiation therAapy for lung tumors. Front Oncol 2018;8:171.

33. Sharma A, Baker S, Duijm M, et al. Prognostic factors for local control and survival for inoperable pulmonary colorectal oligometastases treated with stereotactic body radiotherapy. Radiother Oncol 2020;144:23-29. 